Document downloaded from:

http://hdl.handle.net/10251/145562

This paper must be cited as:

Quiles-Carrillo, L.; Mellinas, C.; Garrigos, M.; Balart, R.; Torres-Giner, S. (11-2). Optimization of microwave-assisted extraction of phenolic compounds with antioxidant activity from carob pods. Food Analytical Methods. 12(11):2480-2490. https://doi.org/10.1007/s12161-019-01596-3

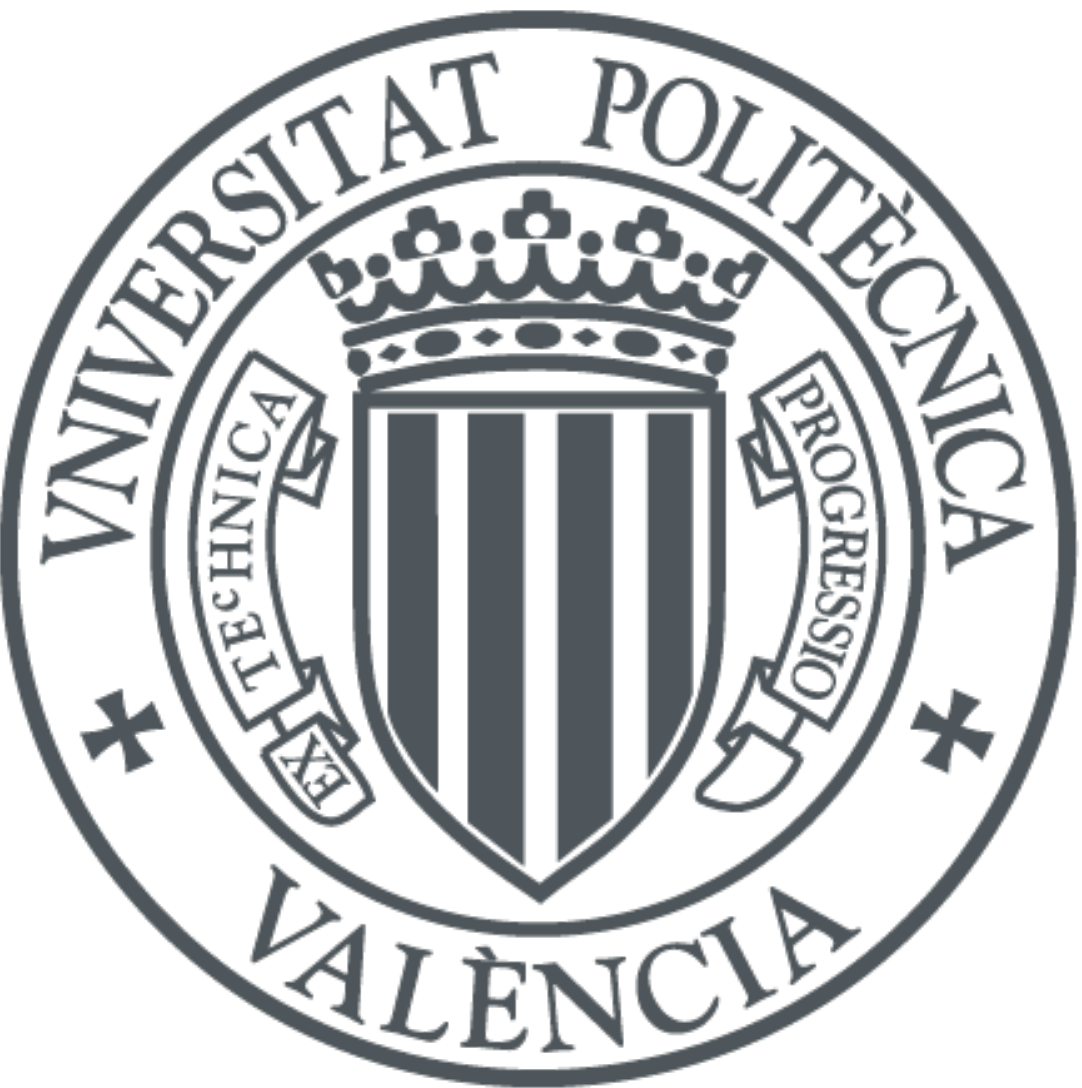

The final publication is available at

https://doi.org/10.1007/s12161-019-01596-3

Copyright Springer-Verlag

Additional Information 


\title{
Optimization of microwave-assisted extraction of phenolic compounds with antioxidant activity from carob pods
}

\author{
L. Quiles-Carrillo ${ }^{(1)^{*}}$, C. Mellinas ${ }^{(2)}$, M.C. Garrigos ${ }^{(2)}$, R. Balart ${ }^{(1)}$, S. Torres-Giner ${ }^{(3)}$ \\ (1) Instituto de Tecnología de Materiales (ITM), Universitat Politècnica de València, \\ Plaza Ferrándiz y Carbonell s/n, Alcoy, (Alicante) \\ *Corresponding author:luiquic1@epsa.upv.es, tel: +34 966528433
}
(2) Analytical Chemistry, Nutrition and Food Sciencies Department, University of Alicante, P.O. Box 99, E-03080, Alicante, Spain

(3) Novel Materials and Nanotechnology Group, Institute of Agrochemistry and Food Technology (IATA), Spanish Council for Scientific Research (CSIC), Calle Catedrático Agustín Escardino Benlloch 7, 46980 Paterna, Spain.

\begin{abstract}
A microwave-assisted extraction (MAE) procedure to obtain phenolic compounds from carob bark was optimized by using response surface methodology. A four-factor, three-level BoxBehnken design with five central points was used to evaluate the influence of temperature, solid-liquid ratio, ethanol concentration and time in carob bark extraction in terms of antioxidant activity (DPPH) and total extraction yield. Optimal extraction conditions were found using $80^{\circ} \mathrm{C}, 35 \%$ (v/v) ethanol, a ratio of $35 \mathrm{~mL} / \mathrm{g}$ and $29.5 \mathrm{~min}$. Total phenolics content (TPC), antioxidant activity (DPPH, FRAP, ABTS), carbohydrates content and main polyphenols composition (HPLC) were determined at optimal conditions. An experimental total yield of $66.5 \%$ was obtained with a TPC value of $33.6 \mathrm{mg}$ GAE/g DW and polysaccharides content of $345.4 \mathrm{mg}$ glucose/g DW. A high antioxidant activity was also shown by the three methods tested. The results showed the potential of carob pods skin as a natural source of phenolic compounds, in particular gallic acid, and the effectiveness of MAE as extraction technique for the revalorization of this agro-food waste.
\end{abstract}

\section{Keywords}

Microwave-assisted extraction, antioxidant activity, carob pods, gallic acid, phenolic compounds, Box-Behnken design 


\section{Introduction}

Currently, the packaging sector is requesting the use of polymer materials of renewable origin due to the great awareness of existing environmental problems. Therefore, the number of both natural and recyclable biopolymer materials that are suitable for their use in the packaging sector is gaining a greater relevance.(Kumar et al., 2017; TĂNASE et al., 2016; Tang et al., 2012). This type of biopolymers, are intended to be combined with active ingredients that may have antioxidant effect for the development of active packaging systems to improve the shelflife of foods(Arrieta et al., 2017). It is also aimed that these compounds could be obtained from agro-food waste(Di Donato et al., 2017; Manousaki et al., 2016), with the aim of increasing the added value of industrial by-products and reducing the total price of the final packaging system. Antioxidants obtained from agro-food waste could greatly enhance the preservation of packaged food in a remarkable way increasing the benefits of this type of products. In this sense, the carob tree fruit is a characteristic product of the Mediterranean area that is currently discarded which is known to have good antioxidant properties, being its valorisation very interesting (Kumazawa et al., 2002).

Carob tree (Ceratonia siliqua L.) is one of the most useful native Mediterranean trees. In producing countries, carob pods have been traditionally used as human and animal food, but currently their main use is related to the extraction of rubber from their seeds.(Owis and ElNaggar, 2016). Carob trees grow around the Mediterranean basin and countries such as Portugal, Spain, Italy, Greece, Turkey, as well as Morocco and several regions of North America. In 2010, world carob production was $163 \mathrm{kt}$, a considerably low amount due to the fact that it is an abandoned plantation. In 2011, the production was doubled reaching $310 \mathrm{kt}$ worldwide, with Spain being the main producer of carob with 135 kt, followed by Italy, Portugal, and Morocco(Vourdoubas and Skoulou, 2017).

Carob pods are characterized by their high content in water-soluble sugars (approximately 40$50 \%$, mainly sucrose) and low content in proteins (3-4\%) and lipids (0.4-0.8\%). Carob pods also contain considerable amounts of polyphenols (Hadrich et al., 2017; Sęczyk et al., 2016), in particular gallic acid (Hadrich et al., 2017). Polyphenols have a wide range of biological properties and, among them, the antioxidant activity is the best known, which can prevent oxidative damage of some biomolecules. Polyphenols have received much attention in recent years due to their ability to act as powerful antioxidants. Polyphenolic compounds are found in the tissues of plants and are important for their growth and development as they provide a defence mechanism against infections and injuries(Karakaya, 2001). In addition, carob pod extracts have been reported to exhibit a very strong antimicrobial activity against several bacteria and fungi(Meziani et al., 2015). 
For the determination of the main polyphenols present in the carob pod and the evaluation of potential applications of these compounds, the optimization of the extraction process of phenolic compounds must be previously carried out(Bai et al., 2010). For this purpose, microwave-assisted extraction (MAE) shows several advantages over conventional extraction techniques such as the reduction of solvent volume and energy consumption used for extraction, obtaining high recoveries and good reproducibility(Filip et al., 2017; Valdés et al., 2015). Nowadays, this technique has been successfully used for the extraction of different food waste matrices such as peanut (Ballard et al., 2010) and tangerine (Hayat et al., 2009) skins and tomato(Pinela et al., 2016), among other wastes with high added value.(Ferreres et al., 2017; Moreira et al., 2017; Rosa et al., 2017) Regarding the extraction of carob pods, some studies have analysed the antioxidant capacity of the obtained extracts but in very few cases MAE has been used(Kumazawa et al., 2002; Makris and Kefalas, 2004; Piñeiro et al., 2017).

The objective of the present study is to revalorize carob bark as a main source of polyphenols giving an added value to this waste. For this purpose, carob beans not suitable for consumption or sale were selected and the seeds were manually extracted and discarded, in order to use as residue only the bark part of the pod. In this work, the optimal MAE conditions to extract polyphenol compounds were determined by using response surface methodology in terms of total yield and antioxidant capacity (DPPH). Independent variables studied included temperature, time, liquid:solvent ratio, and ethanol concentration. Total phenolics (TPC) and carbohydrates content, antioxidant capacity by using FRAP, DPPH and ABTS methods, total yield and quantification of main polyphenols were also determined in the extracts obtained at optimal conditions.

\section{Materials and methods}

\subsection{Raw material and chemical reagents}

Carob pods were obtained from the European carob tree Ceratonia Silicua of the variety "ramillete", which is original of Alicante and Murcia regions (Spain). The obtained bark (Figure 1a) was oven-dried for $48 \mathrm{~h}$ at $40{ }^{\circ} \mathrm{C}$ (MCP Vacuum Casting System, Lubeck, Germany). As a first step, carob bark was manually crushed and then introduced into a centrifugal mill (Maype, Manises, Spain). The obtained powder (Figure 1b) was oven-dried at $40{ }^{\circ} \mathrm{C}$ for $12 \mathrm{~h}$ and grinded in a Mill ZM 200 centrifugal mill (Retsch, Düsseldorf, Germany) at $12000 \mathrm{rpm}$. The milled sample was passed through a $0.25 \mathrm{~mm}$ sieve. This residue (Figure 1c) was oven-dried again during $24 \mathrm{~h}$ at $40^{\circ} \mathrm{C}$.

Quercetin, sodium carbonate, Folin-Ciocalteu reagent (2 N), 2,2-diphenyl-1-picrylhydrazyl (DPPH), ( \pm ) 6-hydroxy-2,5,7,8-tetramethylchromane-2-carboxylic acid (Trolox), ethanol (96\%), 
ethanol (absolute grade), methanol (HPLC grade), acetonitrile (HPLC grade), 2,2'azinobis(3ethylbenzthiazoline)-6-sulfonic acid (ABTS), potassium persulfate, and calcium chloride were purchased from Sigma-Aldrich(Madrid, Spain).

\subsection{Microwave-assisted extraction (MAE) of polyphenols: experimental design}

Extraction of polyphenols was carried out by using a commercial microwaves oven (Milestone flexiWAVE, Shelton, Connecticut, USA). $2 \mathrm{~g}$ of sample was introduced and the microwave agitator was set at $300 \mathrm{rpm}$ during the extraction process. Response surface methodology (RSM) was used to determine the optimal extraction conditions of polyphenols from carob bark. A Box-Behnken design (BBD) was used to determine the effect of four variables which may affect phytochemical contents in plants (Dai and Mumper, 2010): extraction temperature, liquid:solid ratio, ethanol concentration and extraction time. Table 1 shows the selected variables and levels which were set according to experimental limitations and related bibliography(Yang and Zhai, 2010). This design consisted of 29 experiments including five central points to estimate the model's pure error. All experiments were carried out in random order and were performed once. The responses obtained from the experimental design were evaluated in terms of overall yield and antioxidant activity (DPPH method).

After extraction, extracts were centrifuged at $5300 \mathrm{rpm}$ and $4{ }^{\circ} \mathrm{C}$ (Digicen 21, Ortoalresa, Ajalvir, Spain) for 15 min to separate the remaining solid residues from the extraction. The supernatant obtained from the centrifugation process was oven-dried (J.P Selecta S.A, Spain) at $40{ }^{\circ} \mathrm{C}$ for $24 \mathrm{~h}$ and then re-dissolved in $20 \mathrm{~mL}$ ethanol $50 \%(\mathrm{v} / \mathrm{v})$ and frozen at $-20{ }^{\circ} \mathrm{C}$ until analysis was performed. Due to some precipitation after this preservation process, samples were centrifuged again at $5300 \mathrm{rpm}$ and $4{ }^{\circ} \mathrm{C}$ for 15 minutes before analysis to separate any precipitated solid residues.

A multiple linear regression analysis was performed to obtain the regression coefficients following a second-order polynomial model:

$$
Y=\beta_{0}+\sum \beta_{i} X_{i}+\sum \beta_{i i} X_{i}^{2}+\sum \sum \beta_{i j} X_{i} X_{j}
$$

where $\mathrm{Y}$ is the predicted response, $\mathrm{X}$ represents the variables of the system, $\mathrm{i}$ and $\mathrm{j}$ are design variables, $\beta_{0}$ is a constant, $\beta_{\mathrm{i}}$ is the linear coefficient, $\beta \mathrm{ii}$ is the quadratic coefficient, and $\beta_{\mathrm{ij}}$ is the interaction coefficient of variables $i$ and $j$.

The extracts obtained at optimal conditions were also analysed to evaluate the antioxidant performance by FRAP and ABTS methods; total phenolics (TPC) and carbohydrates content. Main polyphenols present in carob extracts were also monitored by HPLC analysis. 


\subsection{Total extraction yield}

The total yield after each extraction was determined as follows:

$$
\text { Extraction yield }(\%)=\frac{W_{\text {ext }}}{W_{0}} \cdot 100
$$

where $\mathrm{W}_{\text {ext }}$ is the mass of the dried extract (g) and $\mathrm{W}_{0}$ is the mass of dried carob bark used for extraction (g).

Samples were weighed by means of an Atilon ATL-224-I analytical balance (Acculab, Bradford, England).

\subsection{Determination of antioxidant capacity}

\subsubsection{DPPH assay}

The 2,2-diphenyl-1-picrylhydrazyl (DPPH) assay was performed according to the methodology described by Sengul Uysal et al. (Uysal et al., 2016). A solution of DPPH $10^{-4} \mathrm{M}$ was prepared by dissolving $0.0039 \mathrm{~g}$ of DPPH radical in $100 \mathrm{~mL}$ of absolute ethanol. This stock solution was daily prepared. $1 \mathrm{~mL}$ of the extract was mixed with $4 \mathrm{~mL}$ of DPPH ethanolic solution and kept in darkness conditions at room temperature for $30 \mathrm{~min}$. The decrease in absorbance was determined at $517 \mathrm{~nm}$ using a Biomate-3 UV-VIS spectrophotometer (Thermospetronic, Mobile, AL, USA).

The antioxidant capacity of the extracts was expressed as percentage of DPPH inhibition by using the following equation:

$$
I(\%)=\frac{A_{0}-A_{1}}{A_{0}} \cdot 100
$$

where $A_{0}$ is the absorbance of the control and $A_{1}$ is the absorbance of the extract after 30 min.

\subsubsection{FRAP assay}

The ferric ion reducing antioxidant power (FRAP) was also determined as described by Sengul Uysal et al. (Uysal et al., 2016). The FRAP reagent was prepared freshly by mixing $300 \mathrm{mM} / \mathrm{L}$ acetate buffer $(\mathrm{pH}=3.6), 10 \mathrm{mM} / \mathrm{L}$ TPTZ and $20 \mathrm{mM} / \mathrm{L}$ ferric chloride in a mixing ratio of 10:1:1 ( $\mathrm{v} / \mathrm{v} / \mathrm{v})$, respectively. Then, $2 \mathrm{~mL}$ of reagent and $200 \mu \mathrm{L}$ of the sample solution were mixed and incubated at $30^{\circ} \mathrm{C}$ for $30 \mathrm{~min}$. The absorbance was then determined at $593 \mathrm{~nm}$. Trolox was used as standard and the results were expressed as mg trolox equivalents per gram dry weight (mg TE/g DW). All samples were analysed in triplicate. 


\subsubsection{ABTS assay}

The antioxidant capacity against 2,2'-azino-bis(3-ethylbenzothiazoline-6-sulphonic acid) (ABTS) was also measured according to the method described by S. Uysal et al. (Uysal et al., 2016). ABTS radical cation was produced directly by reacting $7 \mathrm{mM}$ ABTS solution with $2.45 \mathrm{mM}$ potassium persulfate and allowing the mixture to stand for 12-16 hours in darkness conditions at room temperature. Prior to the assay, the solution was diluted with ethanol to an absorbance of $0.700 \pm 0.02$ at $734 \mathrm{~nm}$. $1 \mathrm{~mL}$ of extract was then added to $2 \mathrm{~mL}$ of ABTS solution and mixed at room temperature for $30 \mathrm{~min}$. The absorbance was determined at $734 \mathrm{~nm}$. ABTS scavenging activity was also expressed as mg TE/g DW and measurements were carried out in triplicate.

\subsection{Total phenolics content (TPC)}

Total phenolics content was determined according to the Folin-Ciocalteu colorimetric assay following the method described by Meziani et al. [13]. $0.5 \mathrm{~mL}$ of extract was mixed with 0.9 $\mathrm{mL}$ of Folin-Ciocalteu reagent and $3.6 \mathrm{~mL}$ of sodium carbonate solution at a concentration of 75 $\mathrm{g} / \mathrm{L}$. The test tubes were stored in darkness conditions at room temperature for $30 \mathrm{~min}$. Absorbance was determined at $765 \mathrm{~nm}$ and the total phenolics content of samples were expressed as milligrams of gallic acid equivalents per g dry weight (mg GAE/g DW). All samples were analysed in triplicate.

\subsection{Phenol-sulfuric acid method}

The basic principle of this method is that carbohydrates, when dehydrated by reaction with concentrated sulfuric acid, can produce furfural derivatives. The subsequent reaction between furfural derivatives and phenol results in a compound with a detectable colour. $2 \mathrm{~mL}$ of the carbohydrate solution (extract) was mixed with $1 \mathrm{~mL}$ of $5 \%$ aqueous solution (v/v) of phenol in a test tube. Subsequently, $5 \mathrm{~mL}$ of concentrated sulfuric acid were rapidly added to the mixture. After $10 \mathrm{~min}$, the mixture was vortexed for $30 \mathrm{~s}$ and placed for 20 minutes in a water bath at room temperature for colour development. Then, absorbance at $490 \mathrm{~nm}$ was recorded. Reference solutions were identically prepared using a calibration curve between 40 and 180 ppm. The results were expressed in mg glucose/g DW.

\subsection{Main polyphenols profile by HPLC analysis}

Prior to the quantification of polyphenols, these were verified using an Agilent highperformance liquid chromatograph model 1100 Series trailer at the same time a variable wavelength UV-visible detector and analyser of ion trap mass spectrometer. The wavelength used was $280 \mathrm{~nm}$ and mass spectrometric detection was performed in the negative ionization 
mode (ESI). After passing through the flow cell of the diode array detector, the column eluate was split (1:10) and the sample was directed to an LCQ ion trap mass spectrometer fitted. Scan range was 50-900 m/z and scan rate, $1 \mathrm{scan} / \mathrm{sec}$. The desolvation temperature was $350{ }^{\circ} \mathrm{C}$. High spray voltage was set at $4500 \mathrm{~V}$. Nitrogen was used as the dry gas at a flow rate of $10 \mathrm{~mL} / \mathrm{min}$.

The identification and quantification of main polyphenols present in carob bark extracts was carried out following the method proposed by SuJung et al. (Hu et al., 2016) with some modifications. $34 \mathrm{mg}$ of dried carob extract was diluted with $50 \mathrm{~mL}$ of ultrapure miliQ water. The diluted solution (1:100 v/v) was analysed for polyphenols content using an Agilent 1260 infinity HPLC system coupled with DAD detector. A BRISA LC2 C18 column (250 mm x 4.6 $\mathrm{mm} \times 5 \mu \mathrm{m}$, Teknokroma) at $30{ }^{\circ} \mathrm{C}$ was used. The mobile phase consisted of two solvents: $0.1 \%$ acetic acid (A) and 100\% acetonitrile (B). The gradient used was: 100\% A (1 min) linearly decreasing to $60 \% \mathrm{~A}$ at $25 \mathrm{~min}(5 \mathrm{~min})$. The flow rate was $0.5 \mathrm{~mL} / \mathrm{min}$ and the injection volume was $20 \mu \mathrm{L}$. Quantification of polyphenols was based on retention times compared with corresponding standards (Sigma-Aldrich, Germany) and their verification were done with Agilent high-performance liquid chromatograph model 1100 Series trailer at the same time a variable wavelength UV-visible detector and Analyzer of ion trap mass spectrometer (Agilent model 1100 Series LC/MSD Trap SL). Analysis was performed in triplicate and mean values were reported. Polyphenols content was calculated from mean peak areas using external calibration method and expressed as mg/g DW.

\subsection{Statistical analysis}

Analysis of variance (ANOVA) was carried out from experimental data and mean values were compared at confidence level of $95 \%(\mathrm{p}<0.05)$ using the Tukey test.

Statgraphics Centurion XVI (Statistical Graphics, Rockville, MD, USA) was used to generate and analyse the results of the BBD. A graphic analysis of the main effects and interactions between the variables was used for results interpretation. Response surface methodology (RSM) was used to determine optimal extraction conditions. Multiple linear regression was performed to obtain regression coefficients of the quadratic polynomial model previously described. Lack of fit, determination coefficient $\left(\mathrm{R}^{2}\right)$ and $\mathrm{F}$ test of the model were evaluated from analysis of variance (ANOVA) at confidence level of $95 \%(\mathrm{p}<0.05)$.

\section{Results}

\subsection{MAE optimization}

The influence of several factors on the the MAE extraction efficiency of polyphenols from carob bark, such as extraction time (min), ethanol concentration (\% v/v), liquid:solid ratio 
(mL/g), and extraction temperature $\left({ }^{\circ} \mathrm{C}\right)$, was studied in terms of total yield (\%) and DPPH inhibition (\%) using surface response methodology. The experimental values selected for each variable in the Box-Behnken design and the responses obtained are given in Table 2.

Figure 2 shows Pareto charts and significant effects at 95\% confidence obtained for the studied responses. The total yield obtained from carob bark ranged from 57.9 to $67.5 \%$. As it can be seen in the Pareto chart, the yield was significantly affected by ethanol concentration and liquid:solid ratio. The ethanol concentration used was the main significant variable influencing extraction yield with a negative effect. So, the yield was increased within the studied levels with the decrease of ethanol concentration. The solvent constitution is one of the most important factors in an extraction process as it could influence the polarity of solvent, being crucial for the solubility of the extracted compounds(Zhao et al., 2018). The presence of water in the solvent could facilitate an increase in extraction yield by improving the swelling of the plant material, which is favourable to increase the surface area of contact between the matrix and the solvent.(Hayat et al., 2009) (Pan et al., 2003) A high concentration of ethanol could interrupt the extraction of phenolic compounds possibly due to a lower penetration of ethanol into the plant matrix through the protective function and a lower solubility of some extractable phenolic compounds.(Tóth et al., 2014) The increase in water content in the solvent composition was statistically influential to improve the extraction yield.(Spigno et al., 2007) In this sense, with decreasing ethanol concentration, not only polyphenols could be extracted but also other compounds such as fructose, glucose or sucrose (present in carob pods) which may result in increasing the total yield (Biner et al., 2007). It was also observed that an increase in the liquid: solid ratio resulted in increasing the total yield. A high solvent volume could accelerate substance transfer and promote solubility by increasing the contact surface of the plant material with the solvent, improving the extraction efficiency(Zhao et al., 2018). In this sense, other authors have shown how a good balance between the amount of ethanol and water can favour a correct extraction of polyphenols(Pan et al., 2003).

Regarding antioxidant capacity, a higher extraction temperature improved the antioxidant capacity which ranged from 68 to $89 \%$ of DPPH inhibition. A high temperature could speed up intermolecular interactions and facilitate molecular motion, which could increase the solubility of the solutes into the solution(Zhao et al., 2018). A significant negative interaction was also observed between time and ethanol concentration, with a lower concentration of ethanol generally improving the antioxidant activity of the extracts. In this sense, a greater amount of ethanol in the concentration generates an increase in the constant dialectic. The increase of this constant increases the microwave temperature, which directly reduces the final yield of the extraction.(Rafiee et al., 2011) Finally, a significant positive interaction between time and 
liquid:solid ratio also affected the antioxidant capacity, increasing DPPH inhibition with higher extraction times and solvent volume.

As a result of the analysis performed, it was decided to focus on the antioxidant capacity as this variable is more directly correlated with the extraction of phenolic compounds compared with the total yield that could be more influenced by the co-extraction of different compounds together with the target polyphenols.

A graphical analysis in terms of response surfaces was performed to study the interactions present in the antioxidant capacity of the obtained extracts (Figure 3). These surface graphics represent in a visual way the interactions between the different factors, giving very valuable information for the analysis and optimization of the extraction. The interaction between time and ethanol concentration is plotted in Figure 3a. As it can be seen, the best results for antioxidant capacity were obtained for a high time and a low ethanol concentration (approaching a 90\% inhibition of DPPH) or for a low time and a high ethanol percentage.

Figure $3 \mathrm{~b}$ shows the interaction between extraction time and liquid:solid ratio, where it can be appreciated that high time values and an intermediate liquid:solid ratio favours obtaining a high percentage of DPPH inhibition. Regarding extraction temperature, the effect of this variable was similar when studying the interaction between the other studied variables, showing an increase in temperature a significant improvement in the antioxidant capacity of the obtained extracts (Figures 3c, 3e and 3f). In particular, the interactions between temperature and liquid:solid ratio or ethanol concentration showed that an intermediate value of these variables together with a high temperature could remarkably improve the antioxidant capacity.

Finally, it can be seen in figure 3d, the interaction between liquid:solid ratio and ethanol concentration with no significant effect on the extraction process under the conditions studied to improve the antioxidant capacity.

The experimental data obtained from all 29 combinations for DPPH was fitted to a second-order polynomial model giving the following equation:

DPPH (\%) $=-95.9163-0.3384 \cdot X_{1}+1.29147 \cdot X_{2}+1.72 \cdot X_{3}+2.86333 \cdot X_{4}-0.00149333 \cdot X_{1}^{2}-$ $0.0216 \cdot \mathrm{X}_{1} \cdot \mathrm{X}_{2}+0.032 \cdot \mathrm{X}_{1} \cdot \mathrm{X}_{3}+0.01 \cdot \mathrm{X}_{1} \cdot \mathrm{X}_{4}-0.000973333 \cdot \mathrm{X}_{2}^{2}+0.0 \cdot \mathrm{X}_{2} \cdot \mathrm{X}_{3}-0.012 \cdot \mathrm{X}_{2} \cdot \mathrm{X}_{4}-$ $0.00733333 \cdot \mathrm{X}_{3}{ }^{2}-0.025 \cdot \mathrm{X}_{3} \cdot \mathrm{X}_{4}-0.00858333 \cdot \mathrm{X}_{4}{ }^{2}$

where $\mathrm{X}_{1}, \mathrm{X}_{2}, \mathrm{X}_{3} \mathrm{y} \mathrm{X}_{4}$ correspond to extraction time, ethanol concentration, liquid:solid ratio and extraction temperature, respectively.

The ANOVA was used for the statistical testing of the model. The non-significance of the lackof-fit tests ( $p=0.2069$ ) verified the suitability of the selected model. The $R^{2}$ value obtained was 
0.69, indicating a certain degree of correlation between experimental and predicted values. The optimal extraction conditions predicted by the model to optimize the extraction of carob bark in terms of antioxidant capacity are: a high extraction time (29.5 $\mathrm{min})$, a low ethanol percentage (35\%), an elevated temperature $\left(80^{\circ} \mathrm{C}\right)$ and an intermediate liquid:solid ratio of $35 \mathrm{~mL} / \mathrm{g}$. These conditions will be tested to verify the degree of confidence of the model.

\subsection{Chemical analysis of extracts at optimal conditions}

The selection of optimal conditions was considered taking into account the conditions predicted by the model and the behaviour observed from the response surface plots. As a result, it was decided to maintain the extraction conditions already predicted but modifying the value of the extraction time (Table 3), which was previously established in 29.5 min (a high value of time). So, it was intended to analyse in a experimental way if the application of a shorter time with the same conditions for the rest of the studied variables could imply some improvement in antioxidant activity. The results obtained for total yield, antioxidant activity (FRAP, DPPH and ABTS methods), TPC and polysaccharides are shown in Table 4.

\subsubsection{Antioxidant capacity}

Table 4 shows the results obtained for antioxidant activity at the different extraction conditions, A and B, which were significantly different for all methods analyzed, obtaining the best results for the extraction performed at B conditions by using a longer extraction time, as predicted by the model. The extended time of microwaves irradiation also resulted in some increase in the extraction yield, obtaining $66.5 \%$ for B extraction which could be considered a high level of extraction capacity at an industrial level for the revalorization of carob waste. It has been reported that, in some cases, polyphenols recovery could be affected by the extension of microwaves extraction time due to some degradation of phenolic compounds.(Song et al., 2011) In this study, however, an increase in extraction time was directly related to an improvement in the antioxidant capacity (Karami et al., 2015); in particular from the results obtained for ABTS and DPPH methods. The used conditions seemed to significantly improve the antioxidant capacity compared to similar carob bean reported extractions.(Roseiro et al., 2013b)

The efficacy of MAE was compared with a conventional Soxhlet extraction (cold water and allowed to stand for $12 \mathrm{~h}$ at $3{ }^{\circ} \mathrm{C}$ and boiled for 10 min with stirring). With this method, a maximum of $20 \%$ to $60 \%$ DPPH inhibition was obtained by using final concentrations of extracts of $10 \mu \mathrm{g} / \mathrm{mL}$ and $50 \mu \mathrm{g} / \mathrm{mL}$, respectively (Kumazawa et al., 2002). Compared to Soxhlet extraction, MAE cost less solvent and required lower extraction temperature and time. 
The variety of the tree is a relevant issue when evaluating the antioxidant capacity. In this sense, no previous study has been found in the literature dealing with the antioxidant capacity of carob pods from the Alicante region of Spain. Worse antioxidant capacities but more polyphenols has been obtained with other pod species. Furthermore, carob pods as industrial product could be a potential source of polyphenol which can possibly substitute the use of synthetic antioxidants, thus improving the functional properties of a range of food products. Studies carried out on carob polyphenols have shown good antioxidant potential in microsomal lipid peroxidation and erythrocyte ghost systems.(Huma et al., 2017)

The experimental results have demonstrated that MAE allows obtaining high values of antioxidant capacity. Even more, it has to be taken into account the origin of the carob pod, and especially its quality, as only poor quality pods and their bark, considered as waste, were used for this study. So, the antioxidant capacity results obtained could be considered very positive for the use of this type of residue as a source of antioxidant compounds.

\subsubsection{Total polyphenols content (TPC)}

Table 5 shows the results obtained for total polyphenols content (TPC) at different extraction conditions. As it can be seen, only by varying the extraction time from 5 to $29.5 \mathrm{~min}$ the amount of polyphenols obtained from the residue was improved by more than $30 \%$, demonstrating also the extraction of phenolic compounds from carob at tested conditions by MAE. A high content of polyphenols were obtained for both extractions when compared to conventional extractions of carobs beans previously reported, showing an average of around $20 \mathrm{mg}$ GAE/g (Turhan et al., 2006). Comparing the obtained results by MAE with other extraction methods such as ultrasound assisted extraction (UAE) and supercritical fluid extraction (SFE) the improvement obtained in this work for TPC was around $100 \%$ and $20 \%$, respectively. (Roseiro et al., 2013a)

In relation to other similar extractions by microwaves, the values obtained have remained below due to the selected conditions. For TPC optimization conditions, values of $70 \mathrm{mg}$ GAE / g were obtained for a time of 4.5 min and an ethanol concentration of 45\%(Huma et al., 2018). This reduction in the TPC values is due to the selection of the factors for a better obtaining of antioxidant properties instead of a greater amount of TPC. If we compare the results obtained with other residues, and in particular the use of the MAE, we can see how the residue studied has very good TPC values. Values of $15.1 \mathrm{mg}$ GAE / g have been obtained for red grape peel and 20.6 mg GAE / g for olive leaves(Makris et al., 2007).

In relation with MAE, Huma et al.(Huma et al., 2018) said that the improvement with other techniques such as UAE and CSE can not be excessively high with high extraction times. This factor is due to the due to degradation of plant cell walls by microwave irradiation as observed 
through scanning electron microscopy. However, the same quantities are extracted in very short time with MAE in comparison with other techniques.

The extraction of polyphenols by using MAE supposes a great opportunity to extract these compounds from the dried residue of carob bark, in a quick and easy way. Depending on the thermal stability of these compounds, they can be further encapsulating to preserve them for further applications. From an industrial point of view, the revalorization of this waste should be optimized, including extraction time, in order to reach a confidence value to optimize the final process.

\subsubsection{Total polysaccharides content}

Although the main objective of this work was not focused on the determination of the total amount of polysaccharides that could be extracted under the tested conditions, it was decided also to evaluate the amount of total polysaccharides present in the obtained extracts due to the complexity of the sample and the visible co-extraction in the process of other compounds such as carob beam gum. Table 4 shows the results obtained for the total polysaccharides content at different extraction conditions. As it can be seen, no significance differences were observed between the polysaccharides content obtained at A and B conditions.

These results show how this type of waste has a large amount of polysaccharides. When analyzing the chemical composition of carob bean pods, Khlifa et al. showed how the polysaccharide compositions of the carob pod are about $50 \%$ of the total amount in its chemical structure.(Khlifa et al., 2013) The residue studied has 35\% polysaccharides in relation to the dry sample. This value indicates a smaller amount of polysaccharides, which can be positive when it comes to obtaining better results of antioxidant capacity.

From the obtained results, it has been shown that this residue contains a large amount of polyphenols, but it is also known that it is a residue rich in sugars, which could be directly related to the obtained results for the carbohydrate analysis.(Biner et al., 2007)

\subsection{HPLC analysis of main polyphenols}

The identification of the type and content of phenolic compounds present in carob bark is essential to determine the potential of the obtained extracts for further applications. Detection and quantification of main phenolic compounds present in carob extract obtained at optimum conditions ( $80^{\circ} \mathrm{C}$; $29.5 \mathrm{~min}$; 35\% ethanol and $35 \mathrm{~mL} / \mathrm{g}$ ratio) was performed by HPLC-DAD. Figure 4 shows polyphenols detected, highlighting the presence of gallic acid as the main polyphenolic compound present in carob extract in accordance with previous works (Almanasrah et al., 2015; Bouli et al., 2010; El Ansari Zineb et al., 2017; Papagiannopoulos et 
al., 2004). Other polyphenols also detected are presented in Table 6. Other authors have reported the presence of other compounds, not detected in this work, such as vanillic acid or rutin (Balaban, 2004). This difference in results could be due to many factors such as environmental differences, extraction procedures, genetic characteristics of the plant, and state of maturity (Stavrou et al., 2018).

\section{Conclusions}

Microwave assisted extraction (MAE) was demonstrated to be an efficient process for the extraction of phenolic compounds with antioxidant activity, in particular gallic acid, from the European carob tree Ceratonia Silicua of the variety "ramillete". A Box Behnken design was used to optimize the MAE experimental conditions by using RSM, obtaining a value of $\mathrm{R}^{2}$ of 0.69. The best extraction conditions were as follows: $80^{\circ} \mathrm{C} ; 29.5 \mathrm{~min} ; 35 \%$ ethanol and $35 \mathrm{~mL} / \mathrm{g}$ ratio. The TPC value and overall yield obtained were $33.6 \pm 0.4 \mathrm{mg}$ GAE/g DW and $66.5 \pm$ $0.4 \%$, respectively. A high concentration of polysaccharides was also co-extracted at optimized conditions. The obtained results from carob tree will contribute to the revalorization of this type of agro-food waste for different applications in the food industry sector, such as the encapsulation of phenolic compounds for functional food or the development of active films. In conclusion, the results from this work have shown the potential of carob barks as a natural source of phenolic compounds with antioxidant performance and the effectiveness of MAE for the reutilization of this agro-food waste.

\section{Funding Information}

This research was supported by the Spanish Ministry of Science, Innovation and Universities (MICIU) programs MAT2017-84909-C2-2-R and AGL2015-63855-C2-1-R and by the EU H2020 project YPACK (reference number 773872). Quiles-Carrillo wants to thank GV for his FPI grant (ACIF/2016/182) and MECD for his FPU grant (FPU15/03812). Torres-Giner also acknowledges MINECO for his Juan de la Cierva contract (IJCI-2016-29675).

\section{Compliance with Ethical Standards}

\section{Conflict of Interest}

Luis Quiles-Carrillo declares that he has no conflict of interest. Cristina Mellinas declares that she has no conflict of interest. Mari Carmen Garrigos declares that she has no conflict of interest. Rafael Balart declares that he has no conflict of interest. Sergio Torres-Giner declares that he has no conflict of interest. 


\section{Ethical approval}

This article does not contain any studies with human participants or animals performed by any of the authors.

\section{Informed Consent}

Not applicable 


\section{References}

Almanasrah, M., Roseiro, L.B., Bogel-Lukasik, R., Carvalheiro, F., Brazinha, C., Crespo, J., Kallioinen, M., Mänttäri, M., Duarte, L.C., (2015). Selective recovery of phenolic compounds and carbohydrates from carob kibbles using water-based extraction. Industrial Crops and Products 70, 443-450.

Arrieta, M., Sessini, V., Peponi, L., (2017). Biodegradable poly (ester-urethane) incorporated with catechin with shape memory and antioxidant activity for food packaging. European Polymer Journal.

Bai, X.L., Yue, T.L., Yuan, Y.H., Zhang, H.W., (2010). Optimization of microwave-assisted extraction of polyphenols from apple pomace using response surface methodology and HPLC analysis. Journal of separation science 33(23-24), 3751-3758.

Balaban, M., (2004). Identification of the main phenolic compounds in wood of Ceratonia siliqua by GC-MS. Phytochemical Analysis: An International Journal of Plant Chemical and Biochemical Techniques 15(6), 385-388.

Ballard, T.S., Mallikarjunan, P., Zhou, K., O'Keefe, S., (2010). Microwave-assisted extraction of phenolic antioxidant compounds from peanut skins. Food chemistry 120(4), 1185-1192.

Biner, B., Gubbuk, H., Karhan, M., Aksu, M., Pekmezci, M., (2007). Sugar profiles of the pods of cultivated and wild types of carob bean (Ceratonia siliqua L.) in Turkey. Food chemistry 100(4), 1453-1455.

Bouli, A.A., Hansali, M., Owen, R.W., (2010). Determination of phenolic composition of carob pods grown in different regions of Morocco. Journal of Natural Products 3.

Dai, J., Mumper, R.J., (2010). Plant phenolics: extraction, analysis and their antioxidant and anticancer properties. Molecules 15(10), 7313-7352.

Di Donato, P., Taurisano, V., Tommonaro, G., Pasquale, V., Jiménez, J.M.S., de Pascual-Teresa, S., Poli, A., Nicolaus, B., (2017). Biological Properties of Polyphenols Extracts from Agro Industry's Wastes. Waste and Biomass Valorization, 1-12.

El Ansari Zineb, B.M., Alain, B., Ahmed, L., (2017). Total Polyphenols and Gallic Acid Contents in Domesticated Carob (Ceratonia siliqua L.) Pods and Leaves. Int. J. Pure App. Biosci 5(4), 22-30.

Ferreres, F., Grosso, C., Gil-Izquierdo, A., Valentão, P., Mota, A.T., Andrade, P.B., (2017). Optimization of the recovery of high-value compounds from pitaya fruit by-products using microwave-assisted extraction. Food chemistry 230, 463-474.

Filip, S., Pavlić, B., Vidović, S., Vladić, J., Zeković, Z., (2017). Optimization of microwave-assisted extraction of polyphenolic compounds from Ocimum basilicum by response surface methodology. Food Analytical Methods 10(7), 2270-2280.

Hadrich, B., Dimitrov, K., Kriaa, K., (2017). Modelling Investigation and Parameters Study of Polyphenols Extraction from Carob (Ceratonia siliqua L.) Using Experimental Factorial Design. Journal of Food Processing and Preservation 41(2).

Hayat, K., Hussain, S., Abbas, S., Farooq, U., Ding, B., Xia, S., Jia, C., Zhang, X., Xia, W., (2009). Optimized microwave-assisted extraction of phenolic acids from citrus mandarin peels and evaluation of antioxidant activity in vitro. Separation and Purification Technology 70(1), 63-70. Hu, S., Kim, B.-Y., Baik, M.-Y., (2016). Physicochemical properties and antioxidant capacity of raw, roasted and puffed cacao beans. Food chemistry 194, 1089-1094.

Huma, Z.E., Jayasena, V., Nasar-Abbas, S.M., Imran, M., Khan, M.K., (2017). Process optimization of polyphenol extraction from carob (Ceratonia siliqua) kibbles using microwaveassisted technique. Journal of Food Processing and Preservation.

Huma, Z.E., Jayasena, V., Nasar-Abbas, S.M., Imran, M., Khan, M.K., (2018). Process optimization of polyphenol extraction from carob (Ceratonia siliqua) kibbles using microwaveassisted technique. Journal of Food Processing and Preservation 42(2), e13450.

Karakaya, S.E., AA Taş, S, (2001). Antioxidant activity of some foods containing phenolic compounds. International journal of food sciences and nutrition 52(6), 501-508. 
Karami, Z., Emam-Djomeh, Z., Mirzaee, H.A., Khomeiri, M., Mahoonak, A.S., Aydani, E., (2015). Optimization of microwave assisted extraction (MAE) and soxhlet extraction of phenolic compound from licorice root. Journal of food science and technology 52(6), 3242-3253.

Khlifa, M., Bahloul, A., Kitane, S., (2013). Determination of chemical composition of carob pod (Ceratonia siliqua L.) and its morphological study. J. Mater. Environ. Sci 4(3), 348-353.

Kumar, N., Kaur, P., Bhatia, S., (2017). Advances in bio-nanocomposite materials for food packaging: a review. Nutrition \& Food Science 47(4).

Kumazawa, S., Taniguchi, M., Suzuki, Y., Shimura, M., Kwon, M.-S., Nakayama, T., (2002). Antioxidant activity of polyphenols in carob pods. Journal of agricultural and food chemistry 50(2), 373-377.

Makris, D.P., Boskou, G., Andrikopoulos, N.K., (2007). Polyphenolic content and in vitro antioxidant characteristics of wine industry and other agri-food solid waste extracts. Journal of Food Composition and Analysis 20(2), 125-132.

Makris, D.P., Kefalas, P., (2004). Carob pods (Ceratonia siliqua L.) as a source of polyphenolic antioxidants. Food technology and biotechnology 42(2), 105-108.

Manousaki, A., Jancheva, M., Grigorakis, S., Makris, D.P., (2016). Extraction of antioxidant phenolics from agri-food waste biomass using a newly designed glycerol-based natural lowtransition temperature mixture: A comparison with conventional eco-friendly solvents. Recycling 1(1), 194-204.

Meziani, S., Oomah, B.D., Zaidi, F., Simon-Levert, A., Bertrand, C., Zaidi-Yahiaoui, R., (2015). Antibacterial activity of carob (Ceratonia siliqua L.) extracts against phytopathogenic bacteria Pectobacterium atrosepticum. Microbial pathogenesis 78, 95-102.

Moreira, M.M., Barroso, M.F., Boeykens, A., Withouck, H., Morais, S., Delerue-Matos, C., (2017). Valorization of apple tree wood residues by polyphenols extraction: Comparison between conventional and microwave-assisted extraction. Industrial Crops and Products 104, 210-220.

Owis, A.I., El-Naggar, E.-M.B., (2016). Identification and quantification of the major constituents in Egyptian carob extract by liquid chromatography-electrospray ionizationtandem mass spectrometry. Pharmacognosy magazine 12(Suppl 1), S1.

Pan, X., Niu, G., Liu, H., (2003). Microwave-assisted extraction of tea polyphenols and tea caffeine from green tea leaves. Chemical Engineering and Processing: Process Intensification 42(2), 129-133.

Papagiannopoulos, M., Wollseifen, H.R., Mellenthin, A., Haber, B., Galensa, R., (2004). Identification and quantification of polyphenols in Carob Fruits (Ceratonia siliqua L.) and derived products by HPLC-UV-ESI/MS n. Journal of agricultural and food chemistry 52(12), 3784-3791.

Pinela, J., Prieto, M., Carvalho, A.M., Barreiro, M.F., Oliveira, M.B.P., Barros, L., Ferreira, I.C., (2016). Microwave-assisted extraction of phenolic acids and flavonoids and production of antioxidant ingredients from tomato: A nutraceutical-oriented optimization study. Separation and Purification Technology 164, 114-124.

Piñeiro, Z., Marrufo-Curtido, A., Vela, C., Palma, M., (2017). Microwave-assisted extraction of stilbenes from woody vine material. Food and Bioproducts Processing 103, 18-26.

Rafiee, Z., Jafari, S., Alami, M., Khomeiri, M., (2011). Microwave-assisted extraction of phenolic compounds from olive leaves; a comparison with maceration. The Journal of Animal \& Plant Sciences 21(4), 738-745.

Rosa, R., Tassi, L., Orteca, G., Saladini, M., Villa, C., Veronesi, P., Leonelli, C., Ferrari, E., (2017). Process intensification by experimental design application to microwave-assisted extraction of phenolic compounds from Juglans regia L. Food Analytical Methods 10(3), 575-586.

Roseiro, L.B., Duarte, L.C., Oliveira, D.L., Roque, R., Bernardo-Gil, M.G., Martins, A.I., Sepúlveda, C., Almeida, J., Meireles, M., Gírio, F.M., (2013a). Supercritical, ultrasound and conventional extracts from carob (Ceratonia siliqua L.) biomass: Effect on the phenolic profile and antiproliferative activity. Industrial Crops and Products 47, 132-138. 
Roseiro, L.B., Tavares, C.S., Roseiro, J.C., Rauter, A.P., (2013b). Antioxidants from aqueous decoction of carob pods biomass (Ceretonia siliqua L.): Optimisation using response surface methodology and phenolic profile by capillary electrophoresis. Industrial Crops and Products 44, 119-126.

Sęczyk, Ł., Świeca, M., Gawlik-Dziki, U., (2016). Effect of carob (Ceratonia siliqua L.) flour on the antioxidant potential, nutritional quality, and sensory characteristics of fortified durum wheat pasta. Food chemistry 194, 637-642.

Song, J., Li, D., Liu, C., Zhang, Y., (2011). Optimized microwave-assisted extraction of total phenolics (TP) from Ipomoea batatas leaves and its antioxidant activity. Innovative food science \& emerging technologies 12(3), 282-287.

Spigno, G., Tramelli, L., De Faveri, D.M., (2007). Effects of extraction time, temperature and solvent on concentration and antioxidant activity of grape marc phenolics. Journal of food engineering 81(1), 200-208.

Stavrou, I.J., Christou, A., Kapnissi-Christodoulou, C.P., (2018). Polyphenols in carobs: A review on their composition, antioxidant capacity and cytotoxic effects, and health impact. Food chemistry.

TĂNASE, E.E., POPA, V.I., POPA, M.E., RÂPĂ, M., POPA, O., (2016). Biodegradation study of some food packaging biopolymers based on PVA. Bulletin UASVM Animal Science and Biotechnologies 73, 1.

Tang, X., Kumar, P., Alavi, S., Sandeep, K., (2012). Recent advances in biopolymers and biopolymer-based nanocomposites for food packaging materials. Critical reviews in food science and nutrition 52(5), 426-442.

Tóth, M.E., Vígh, L., Sántha, M., (2014). Alcohol stress, membranes, and chaperones. Cell Stress and Chaperones 19(3), 299-309.

Turhan, I., Tetik, N., Aksu, M., Karhan, M., Certel, M., (2006). Liquid-solid extraction of soluble solids and total phenolic compounds of carob bean (Ceratonia siliqua L.). Journal of food process engineering 29(5), 498-507.

Uysal, S., Zengin, G., Aktumsek, A., Karatas, S., (2016). Chemical and biological approaches on nine fruit tree leaves collected from the Mediterranean region of Turkey. Journal of Functional Foods 22, 518-532.

Valdés, A., Vidal, L., Beltrán, A., Canals, A., Garrigós, M.C., (2015). Microwave-assisted extraction of phenolic compounds from almond skin byproducts (prunus amygdalus): A multivariate analysis approach. Journal of agricultural and food chemistry 63(22), 5395-5402.

Vourdoubas, J., Skoulou, V.K., (2017). Possibilities of Upgrading Solid Underutilized Lingocellulosic Feedstock (Carob Pods) to Liquid Bio-fuel: Bio-ethanol Production and Electricity Generation in Fuel Cells-A Critical Appraisal of the Required Processes. Studies in Engineering and Technology 4(1), 25-34.

Yang, Z., Zhai, W., (2010). Optimization of microwave-assisted extraction of anthocyanins from purple corn (Zea mays L.) cob and identification with HPLC-MS. Innovative food science \& emerging technologies 11(3), 470-476.

Zhao, C.-N., Zhang, J.-J., Li, Y., Meng, X., Li, H.-B., (2018). Microwave-assisted extraction of phenolic compounds from Melastoma sanguineum fruit: Optimization and identification. Molecules 23(10), 2498. 


\section{Figures}

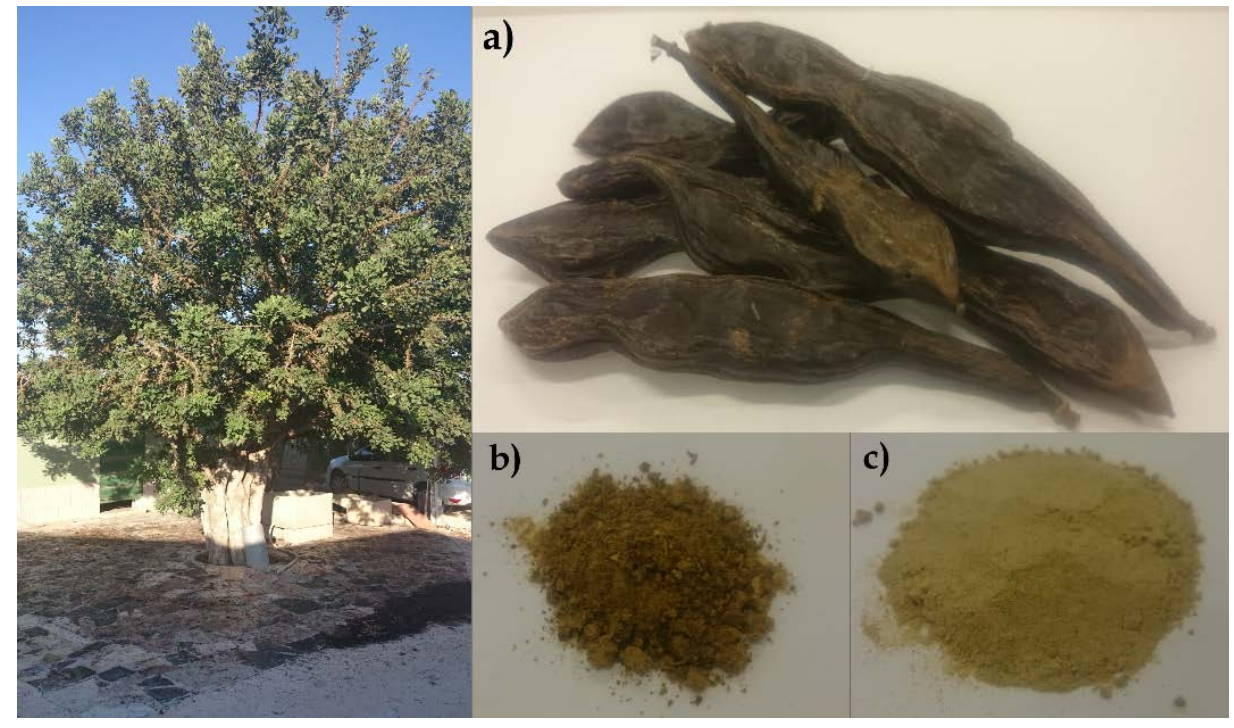

Figure 1. Carob pods (a) and grinding process to obtain carob flour (b and c). 


\section{Yield Extraction}
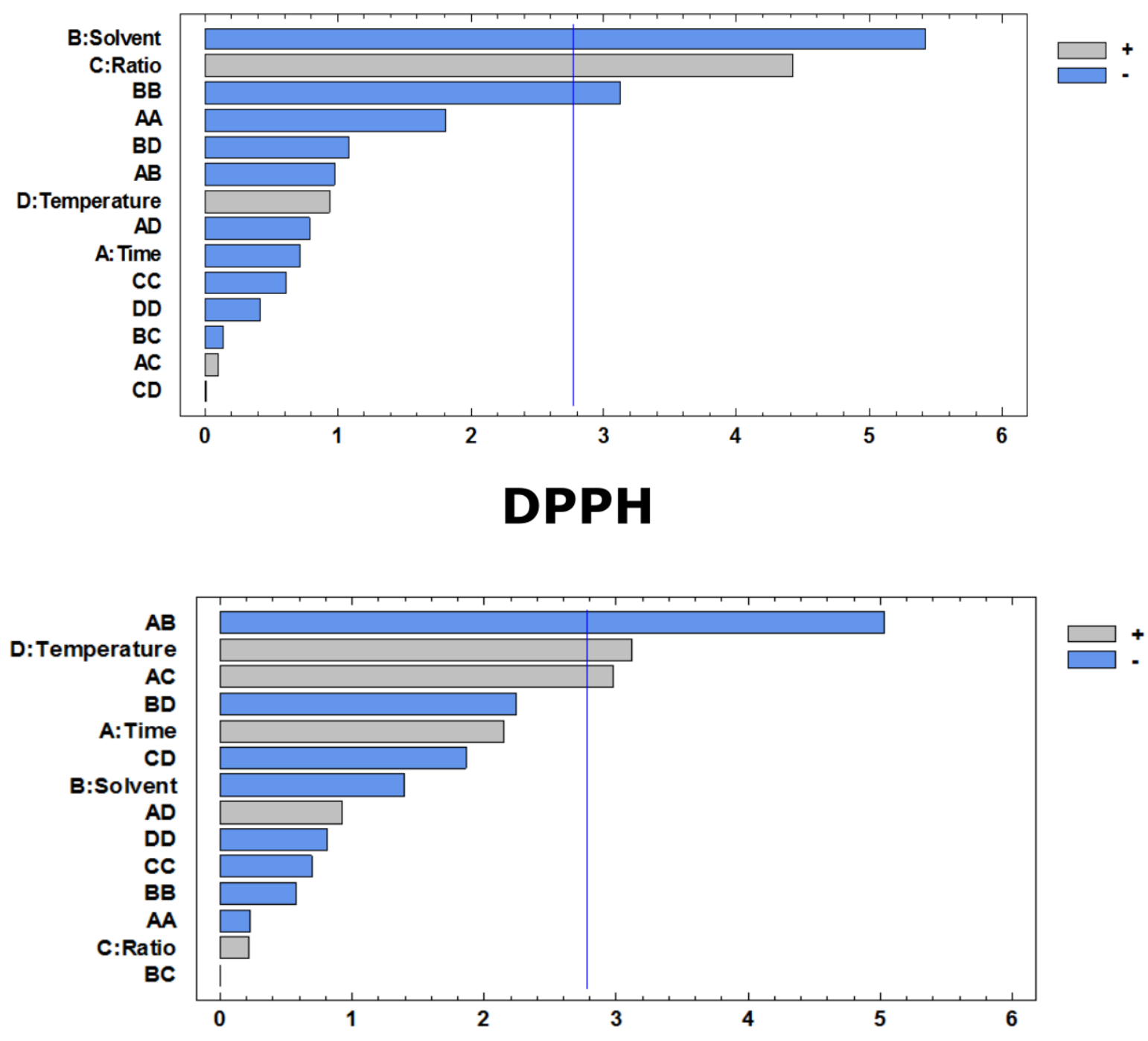

Figure 2. Pareto charts obtained for extraction yield and antioxidant capacity (DPPH) of carob bark. 

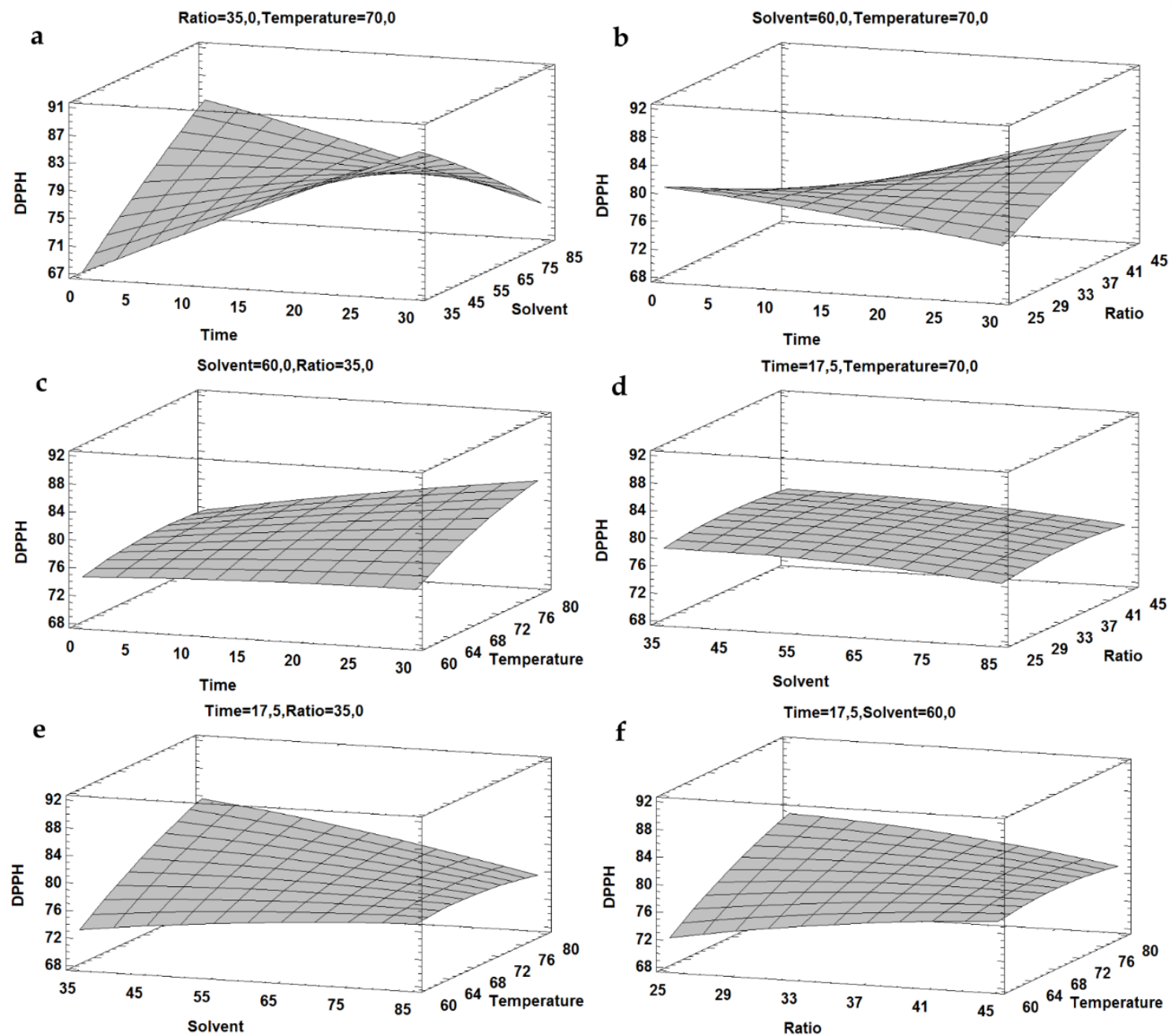

Figure 3. Response surface plots showing the effect of the studied variables on the antioxidant capacity (DPPH) of extracts obtained from carob bark. 


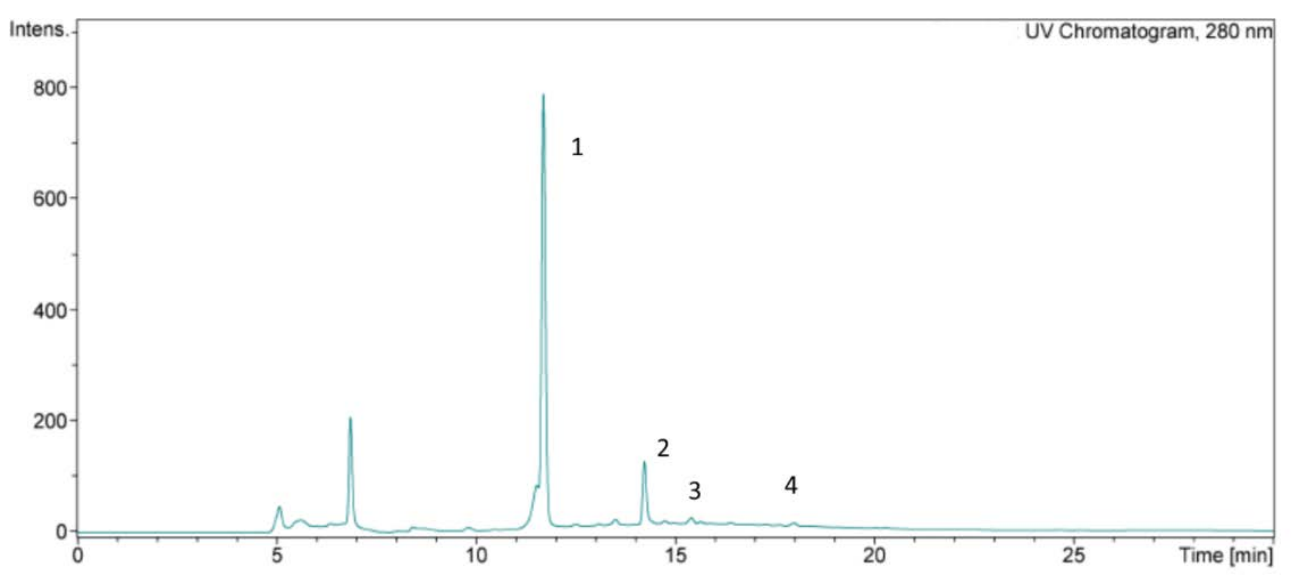

Figure 4. Chromatogram of main compounds found in carob (Table 6) at $280 \mathrm{~nm}$. 


\section{Tables}

Table 1. Independent variables and selected levels used in the BBD for polyphenols extraction from carob bark.

\begin{tabular}{|c|c|c|c|}
\hline Factors & $\mathbf{- 1}$ & $\mathbf{0}$ & $\mathbf{+ 1}$ \\
\hline Temperature ( $\mathbf{(}^{\mathbf{C}} \mathbf{)}$ & 60 & 70 & 80 \\
\hline Ratio (mL/g) & 25 & 35 & 45 \\
\hline Concentration (\%) & 35 & 60 & 85 \\
\hline Time (min) & 5.0 & 17.5 & 30.0 \\
\hline
\end{tabular}


Table 2. Box-Behnken Experimental Design used and MAE responses obtained from carob bark.

\begin{tabular}{|c|c|c|c|c|c|c|}
\hline Experiment & $\begin{array}{l}\text { Time } \\
\text { (min) }\end{array}$ & $\begin{array}{c}\text { Ethanol } \\
(\%)\end{array}$ & $\begin{array}{l}\text { Ratio } \\
(\mathrm{mL} / \mathrm{g})\end{array}$ & $\begin{array}{c}\text { Temperature } \\
\left({ }^{\circ} \mathrm{C}\right)\end{array}$ & Yield (\%) & $\begin{array}{c}\text { DPPH } \\
(\%)\end{array}$ \\
\hline 1 & 30 & 35 & 35 & 70 & 62.5 & 87 \\
\hline 2 & 5 & 60 & 35 & 60 & 62.1 & 79 \\
\hline 3 & 30 & 60 & 35 & 80 & 63.7 & 81 \\
\hline 4 & 5 & 35 & 35 & 70 & 63.4 & 68 \\
\hline 5 & 17.5 & 35 & 25 & 70 & 62.7 & 78 \\
\hline 6 & 17.5 & 85 & 35 & 80 & 60.0 & 74 \\
\hline 7 & 17.5 & 85 & 25 & 70 & 57.9 & 81 \\
\hline 8 & 17.5 & 85 & 45 & 70 & 62.4 & 79 \\
\hline 9 & 17.5 & 60 & 45 & 60 & 64.6 & 80 \\
\hline 10 & 30 & 60 & 25 & 70 & 61.8 & 77 \\
\hline 11 & 30 & 60 & 35 & 60 & 63.4 & 75 \\
\hline 12 & 17.5 & 60 & 35 & 70 & 62.5 & 77 \\
\hline 13 & 5 & 60 & 35 & 80 & 64.5 & 80 \\
\hline 14 & 17.5 & 35 & 35 & 80 & 66.1 & 89 \\
\hline 15 & 5 & 60 & 25 & 70 & 61.7 & 79 \\
\hline 16 & 17.5 & 60 & 35 & 70 & 64.2 & 80 \\
\hline 17 & 17.5 & 60 & 35 & 70 & 65.1 & 81 \\
\hline 18 & 17.5 & 60 & 35 & 70 & 64.1 & 75 \\
\hline 19 & 17.5 & 60 & 25 & 60 & 62.1 & 71 \\
\hline 20 & 17.5 & 35 & 45 & 70 & 67.5 & 76 \\
\hline 21 & 17.5 & 60 & 35 & 70 & 66.1 & 81 \\
\hline 22 & 30 & 85 & 35 & 70 & 58.1 & 73 \\
\hline 23 & 30 & 60 & 45 & 70 & 65.1 & 84 \\
\hline 24 & 17.5 & 85 & 35 & 60 & 60.8 & 71 \\
\hline 25 & 17.5 & 35 & 35 & 60 & 64.0 & 74 \\
\hline 26 & 17.5 & 60 & 25 & 80 & 62.3 & 78 \\
\hline 27 & 5 & 85 & 35 & 70 & 61.6 & 81 \\
\hline 28 & 17.5 & 60 & 45 & 80 & 64.8 & 77 \\
\hline 29 & 5 & 60 & 45 & 70 & 64.7 & 70 \\
\hline
\end{tabular}


Table 3. Optimal conditions found for polyphenols extraction from carob bark.

\begin{tabular}{|c|c|c|c|c|}
\hline \multirow{2}{*}{$\begin{array}{c}\text { Optimal } \\
\text { extraction } \\
\text { conditions }\end{array}$} & \multicolumn{4}{|c|}{ Factors } \\
\cline { 2 - 5 } & Temperature ( $\left.{ }^{\mathbf{0}} \mathbf{C}\right)$ & Ratio (mL/g) & Ethanol (\%) & Time(min) \\
\hline A & 80 & 35 & 35 & 5 \\
\hline B & 80 & 35 & 35 & 29.5 \\
\hline
\end{tabular}


Table 4. Results obtained at different extraction times to optimize the extraction of carob bark

\begin{tabular}{|l|c|c|}
\hline Optimal extraction conditions & A & B \\
\hline Extraction yield (\%) & $65.2 \pm 0.5^{\mathrm{a}}$ & $66.5 \pm 0.4^{\mathrm{b}}$ \\
\hline FRAP( $\mu$ MTEs/g DW) & $85.0 \pm 2.0^{\mathrm{a}}$ & $107.2 \pm 1.7^{\mathrm{b}}$ \\
\hline DPPH (\% inhibition) & $73.0 \pm 1.0^{\mathrm{a}}$ & $82.0 \pm 1.0^{\mathrm{b}}$ \\
\hline ABTS (mg TE/g DW) & $14.9 \pm 0.5^{\mathrm{a}}$ & $22.0 \pm 1.0^{\mathrm{b}}$ \\
\hline TPC (mg GAE/g DW) & $25.6 \pm 0.3^{\mathrm{a}}$ & $33.6 \pm 0.4^{\mathrm{b}}$ \\
\hline Polysaccharides (mg glucose/g DW) & $336.4 \pm 11.1^{\mathrm{a}}$ & $345.4 \pm 14.3^{\mathrm{b}}$ \\
\hline
\end{tabular}

$(\mathrm{n}=3)$ mean \pm SD // Different superscripts within the same row indicate significant differences between values $(p<0.05)$ 
Table 6. Quantification of main compounds found in carob by HPLC.

\begin{tabular}{|c|c|c|c|}
\hline & Time $(\mathrm{min})$ & Detected compound & $\mathrm{mg} / \mathrm{g} \mathrm{DW}$ \\
\hline $\mathbf{1}$ & 11.5 & Gallic acid & $25.37 \pm 0.26$ \\
\hline $\mathbf{2}$ & 14.2 & Cathequin & $18.70 \pm 0.19$ \\
\hline $\mathbf{3}$ & 15.4 & 4-hidroxybenzoic acid & $2.59 \pm 0.10$ \\
\hline $\mathbf{4}$ & 16.8 & Epigallocathequin & $1.10 \pm 0.09$ \\
\hline \multicolumn{3}{|c}{$*$ (mean $\mathbf{s d}, \mathbf{n = 3})$}
\end{tabular}

